\title{
ON RELATED VECTOR FIELDS OF CAPILLARY SURFACES
}

\author{
AFET K. ÖZOK \\ Department of Mathematics \\ Istanbul University \\ Faculty of Science \\ Vezneciler-Istanbul, Turkey
}

(Received August 1, 1980)

ABSTRACT. In this paper, some necessary and sufficient conditions are given for the related vector fields of capillary surfaces to be Killing, conformal Killing, and homothetic conformal Killing vectors in the n-dimensional domain $\Omega$, and a construction of capillary surfaces is also given by means of the related vector fields.

KEY WORDS AND PHRASES. Vector fields, capillary surfaces, Killing, conformal Killing, and homothetic conformal Killing vectors.

1980 MATHEMATICS SUBJECT CLASSIFICATION CODES. $\quad 53 \mathrm{C}$

1. INTRODUCTION.

In the non-parametric "capillary problem" in the absence of gravity, one seeks 
a surface $s: u=u\left(x_{1}, \ldots, x_{n}\right)$, of constant mean curvature $H$, defined over a region $\Omega$ in $n$-dimensional Euclidean space $n \geq 2$, such that $s$ meets vertical cylinder walls $z$ over $\Sigma=\partial \Omega$ in a (prescribed) constant angle $\gamma$. One is led to the equation

$$
\operatorname{div} \frac{\nabla u}{\sqrt{1+|\nabla u|^{2}}}=\mathrm{nH}
$$

in $\Omega$, with boundary condition

$$
\mathrm{v} \cdot \frac{\nabla \mathrm{u}}{\sqrt{1+|\nabla \mathrm{u}|^{2}}}=\cos \gamma
$$

on $\Sigma$. Here $v$ is the exterior unit normal on $\Sigma$. (For physical and geometrical background information, see [1].)

It is shown by R. Finn [2] that, in any situation for which the problem $[(1.1),(1.2)]$ has a solution, there exists a vector field

$$
\omega(x)=\frac{\nabla u}{(\cos \gamma) \sqrt{1+|\nabla u|^{2}}}
$$

in $\Omega$, satisfying

$$
\begin{array}{ll}
\operatorname{div} \omega=\frac{\Sigma}{\Omega} & \text { in } \Omega \\
v \cdot \omega=1 & \text { on } \Sigma \\
\underset{\Omega}{\operatorname{ubb}|\omega| \leq \frac{1}{\cos \gamma} .}
\end{array}
$$

The symbols $\Omega, \Sigma$ are used here to denote alternatively both a set and its measure.

So we have a Euclidean vector field in an $n$-dimensional domain $\Omega, n \geq 2$. We consider $\Omega$ as a part of n-dimensional Euclidean space $E_{n}$, with the fundamental positive definite metric 


$$
d s^{2}=\sum_{1, j=1}^{n} \delta_{1 j} d x_{1} d x_{j}
$$

covered by any system of coordinate neighborhoods $\left(x_{1}\right)$. It is well known that some special kind of vectors (for example, Killing, conformal Killing, homothetic conformal killing vectors) define motions (isometries) in a metric space [3], [4], [5], [6], [7]. The purpose of this paper is to determine for which kind of "capillary surfaces" the related vector field (1.3) in $\Omega$ defines the above mentioned motions. And in Section 4, a construction of capillary surfaces is given by means of related vector fields.

2. THE CONDITIONS FOR THE VECTOR FIELD (1.3) TO BE A KILLING VECTOR IN $\Omega$.

The vector field (1.3) can be written in the form

$$
\omega_{1}(x)=\frac{u_{i}(x)}{(\cos \gamma) \sqrt{1+u_{1}^{2}+\cdots+u_{n}^{2}}}, \quad(i=1, \ldots, n)
$$

where $u_{i}=\partial u / \partial x_{i}$. On the other hand, the condition for the vector field $\omega_{i}(x)$ to be a Killing vector is

$$
\frac{\partial \omega_{1}}{\partial x_{j}}+\frac{\partial \omega_{1}}{\partial x_{i}}=0, \quad(i=1, \ldots, n)
$$

and consequently

$$
\operatorname{div} \omega=\sum_{i=1}^{n} \frac{\partial \omega_{i}}{\partial x_{i}}=0
$$

i) In the case where the problem $[(1.1),(1.2)]$ has a solution $\mathrm{H}=$ const. $\neq 0$, we have by virtue of equation (1.1)

$$
\operatorname{div} \frac{\nabla u}{\sqrt{1+|\nabla u|^{2}}}=\operatorname{div}[(\cos \gamma) \omega]=\mathrm{nH}
$$


and hence

$$
\operatorname{div} \omega=\frac{\mathrm{nH}}{\cos \gamma} \neq 0 \text {. }
$$

Thus we obtain

THEOREM 1. If the problem $[(1.1),(1.2)]$ has a solution $\mathrm{H}=$ const. $\neq 0$, then the related vector field (1.3) in $\Omega$ cannot be a killing vector. Thus the vector field $\omega_{i}(x)$ cannot generate a local one-parameter group of motions in $\Omega$.

ii) If the problem $[(1.1),(1.2)]$ has a solution $H=0$, i.e., if we have a minimal capillary surface, by virtue of equation (2.1), we find from (2.2) the following conditions

$$
\left(1+\sum_{k=1}^{n} u_{k}^{2}\right) u_{i i}-\sum_{k=1}^{n} u_{i k} u_{i} u_{k}=0, \quad(i=1, \ldots, n)
$$

and

$$
2\left(1+\sum u_{k}^{2}\right) u_{i j}-\sum_{k=1}^{n}\left(u_{i k} u_{j} u_{k}+u_{j k} u_{i} u_{k}\right)=0, \quad(i \neq j, i, j=1, \ldots, n)
$$

where

$$
u_{k}=\frac{\partial u}{\partial x_{k}} \quad \text { and } \quad u_{k i}=u_{i k}=\frac{\partial^{2} u}{\partial x_{k} \partial x_{i}}=\frac{\partial^{2} u}{\partial x_{i} \partial x_{k}}
$$

On the other hand, for the surface $s: u=u\left(x_{1}, \ldots, x_{n}\right)$, the coefficients of the first and second fundamental forms are defined

$$
g_{i i}=1+u_{i}^{2}, \quad g_{i j}=u_{i j} u_{j} ; \quad d_{i j}=\frac{u_{i j}}{\sqrt{1+u_{1}^{2}+\cdots+u_{n}^{2}}} .
$$

So equations (2.5) and (2.6) take the forms respectively:

$$
\left(2-n+\sum_{k=1}^{n} g_{k k}\right) d_{i i}=\sum_{k=1}^{n} g_{i k} d_{i k}, \quad(i=1, \ldots, n)
$$

and 


$$
2\left(2-n+\sum_{k=1}^{n} g_{k k}\right) d_{i j}=\sum_{k=1}^{n}\left(g_{i k} d_{j k}+g_{j k} d_{i k}\right) \quad(i \neq j ; i, j=1, \ldots, n) .
$$

The above equations (2.8) and (2.9) can also be put in the form

$$
\frac{\sum_{k=1}^{n} g_{i k} d_{i k}}{d_{i i}}=\frac{\sum_{k=1}^{n}\left(g_{i k} d_{j k}+g_{i k} d_{i k}\right)}{2 d_{i j}}=\left(2-n+\sum_{k=1}^{n} g_{k k}\right) \quad(i \neq j ; i, j=1, \ldots, n) \text {. }
$$

Hence we have

THEOREM 2. If the problem $[(1.1),(1.2)]$ has a solution $H=0$, then the necessary and sufficient condition for the related vector field (1.3) to be a Killing vector in the $n$-dimensional domain $\Omega$ is

$$
\frac{\sum_{k=1}^{n} g_{i k} d_{i k}}{d_{i i}}=\frac{\sum_{k=1}^{n}\left(g_{i k} d_{j k}+g_{j k} d_{i k}\right)}{2 d_{i j}}=\left(2-n+\sum_{k=1}^{n} g_{k k}\right) \quad(i \neq j ; i, j=1, \ldots, n)
$$

where $g_{i j}$ and $d_{i j}$ are the coefficients of the first and second fundamental forms of the minimal capillary surface $u(x)$.

THEOREM 3. For the minimal capillary surfaces for which the coefficients of the first and second fundamental forms satisfy the condition $(2.10)$, the related vector field (1.3) generates a local one-parameter group of motions in the n-dimensional domain $\Omega$.

APPLICATION 1 . In the case $n=2$, we find from equation (2.10)

$$
g_{22} d_{11}=g_{12} d_{12}, \quad g_{11} d_{22}=g_{12} d_{12}
$$

and

$$
\left(g_{11}+g_{22}\right) d_{12}=\left(d_{11}+d_{22}\right) g_{12} .
$$

So we have 


$$
\frac{d_{11}}{g_{11}}=\frac{d_{22}}{g_{22}}=\frac{d_{12}}{g_{12}}=\rho
$$

Hence, the minimal capillary surface must be a totally umbilical surface. But the sphere is the only surface, all points of which are umbilics. So, if $n=2$, the minimal capillary surface does not exist such that the related vector field (1.3) is a killing vector in $\Omega$. Then from this result and Theorem 1 we obtain

THEOREM 4. In the case $\mathrm{n}=2$, in any situation for which the problem [(1.1), (1.2)] has a solution, the related vector field (1.3) in $\Omega$ cannot be a Killing vector; thus the vector field $\omega(x)$ cannot generate a local one-parameter group of motions in $\Omega$.

3. THE CONDITIONS FOR THE VECTOR FIELD (1.3) TO BE A CONFORMAL KILLING AND HOMOTHETIC CONFORMAL KILLING VECTOR IN $\Omega$.

The condition for the vector field $\omega_{i}(x)$ to be a conformal killing vector is

$$
\frac{\partial \omega_{i}}{\partial x_{j}}+\frac{\partial \omega_{j}}{\partial x_{i}}=2 \varphi \delta_{i j}, \quad(i, j=1, \ldots, n)
$$

where $\varphi$ is a certain scalar function. Since equation (3.1) gives

$$
\varphi=\frac{1}{n} \operatorname{div} \omega
$$

substituting equation (2.4) into (3.2), we find

$$
\varphi=\frac{H}{\cos \gamma}=\alpha=\text { constant }
$$

(If $H=0,(3.3)$ gives $\varphi=0$ and equation (3.1) takes the form (2.2).) Thus, according to the definition of the homothetic conformal Killing vectors [8], the vector field (3.1) can be a homothetic conformal Killing vector.

By virtue of equation (2.1), we find from equation (3.1) the following conditions : 


$$
\left(1+\sum_{k=1}^{n} u_{k}^{2}\right)\left(u_{i i}-u_{j j}\right)=\sum_{k=1}^{n}\left(u_{i k} u_{i} u_{k}-u_{j k} u_{j} u_{k}\right) \quad(i, j=1, \ldots, n)
$$

and

$$
2\left(1+\sum_{k=1}^{n} u_{k}^{2}\right) u_{i j}=\sum_{k=1}^{n}\left(u_{i k} u_{j} u_{k}+u_{j k} u_{i} u_{k}\right) \quad(i \neq j ; i, j=1, \ldots, n)
$$

Substituting (2.7) into (3.4) and (3.5), we find

$$
\left(2-n+\sum_{k=1}^{n} g_{k k}\right)\left(d_{i i}-d_{j j}\right)=\sum_{k=1}^{n}\left(g_{i k} d_{i k}-g_{j k} d_{j k}\right) \quad(i, j=1, \ldots, n)
$$

and

$$
2\left(2-n+\sum_{k=1}^{n} g_{k k}\right) d_{i j}=\sum_{k=1}^{n}\left(g_{i k} d_{j k}+g_{j k} d_{i k}\right) \quad(i \neq j, i, j=1, \ldots, n) .
$$

Hence

$$
\begin{array}{r}
\frac{\sum_{k=1}^{n}\left(g_{i k} d_{i k}-g_{j k} d_{j k}\right)}{\left(d_{i i}-d_{j j}\right)}=\frac{\sum_{k=1}^{n}\left(g_{i k} d_{j k}+g_{j k} d_{i k}\right)}{2 d_{i j}}=\left(2-n+\sum_{k=1}^{n} g_{k k}\right) \\
\quad(i \neq j ; i, j=1, \ldots, n) .
\end{array}
$$

Thus we have

THEOREM 5. The necessary and sufficient condition for the vector field (1.3) to be a homothetic conformal killing vector in the n-dimensional domain $\Omega$ is

$$
\frac{\sum_{k=1}^{n}\left(g_{i k} d_{i k}-g_{j k} d_{j k}\right)}{\left(d_{i i}-d_{j j}\right)}=\frac{\sum_{k=1}^{n}\left(g_{i k} d_{j k}+g_{j k} d_{i k}\right)}{2 d_{i j}}=\left(2-n+\sum_{k=1}^{n} g_{k k}\right) \quad(i \neq j ; i, j=1, \ldots, n)
$$

where $g_{i j}$ and $d_{i j}$ are the coefficients of the first and second fundamental forms of the capillary surface $u(x)$. 
THEOREM 6. For the capillary surfaces for which the coefficients of the first and second fundamental forms satisfy the condition (3.8), the related vector field (1.3) generates a local one-parameter group of homothetic motions in the $\mathrm{n}$-dimensional domain $\Omega$.

APPLICATION 2. In the case $\mathrm{n}=2$, we obtain from equation (3.8)

$$
g_{11} d_{22}=g_{22} d_{11}
$$

and

$$
\left(g_{11}+g_{22}\right) d_{12}=\left(d_{11}+d_{22}\right) g_{12} \text {. }
$$

So we have

$$
\frac{d_{11}}{g_{11}}=\frac{d_{22}}{g_{22}}=\frac{d_{12}}{g_{12}}=\rho \text {. }
$$

Thus we have

THEOREM 7. In the case $n=2$, the only capillary surface for which the related vector field (1.3) is a homothetic conformal killing vector in $\Omega$ is a sphere.

THEOREM 8. In the case $\mathrm{n}=2$, if the solution of the problem $[(1,1),(1.2)]$ is a sphere, the related vector field (1.3) generates a local one-parameter group of homothetic motions in $\Omega$.

Integrating equation (3.1), we can also find the form of a homothetic conformal Killing vector:

$$
\omega_{i}(x)=\alpha x_{i}+\sum_{j=1}^{n} \alpha_{i j} x_{j}+\alpha_{i} \quad(i=1, \ldots, n)
$$

where the $\alpha^{\prime} s$ are constants, subject to the conditions 


$$
\alpha=\varphi=\frac{H}{\cos \gamma} \quad \text { and } \quad \alpha_{i j}=-\alpha_{j i} .
$$

APPLICATION 3. Let the lower hemisphere, centered at the point $M\left(m_{1}, \ldots, m_{n+1}\right)$ with radius $r$, be a solution of the problem [(1.1), (1.2)]

$$
u(x)=m_{n+1}-\left[r^{2}-\left(x_{1}-m_{1}\right)^{2}-\cdots-\left(x_{n}-m_{n}\right)^{2}\right]^{\frac{1}{2}} .
$$

Since

$$
u_{1}=\frac{\left(x_{1}-m_{1}\right)}{m_{n+1}-u}, \quad(i=1, \ldots, n)
$$

and

$$
1+u_{1}^{2}+\cdots+u_{n}^{2}=\frac{r^{2}}{\left(m_{n+1}-u\right)^{2}},
$$

from equation (2.1) we find

$$
\omega_{i}(x)= \pm\left[\frac{1}{r \cos \gamma} x_{i}-\frac{m_{i}}{r \cos \gamma}\right], \quad(i=1, \ldots, n)
$$

where $r, \cos \gamma$ and $m_{i}$ are constants. So, $\omega_{i}(x)$ has the form (3.9).

Thus we have

THEOREM 9. For an arbitrary $\mathrm{n}$, if the solution of the problem $[(1.1),(1.2)]$ is a sphere, the related vector field (1.3) is a homothetic conformal Killing vector in the $\mathrm{n}$-dimensional domain $\Omega, \mathrm{n} \geq 2$.

And we have also

THEOREM 10. For an arbitrary $n$, if the solution of the problem $[(1.1),(1.2)]$ is a sphere, the related vector field (1.3) generates a local one-parameter group of homothetic motions in the n-dimensional domain $\Omega, \mathrm{n} \geq 2$. 
4. CONSTRUCTION OF CAPILLARY SURFACES BY MEANS OF RELATED VECTOR FIELDS.

i) If we have a homothetic conformal Killing vector of the form

$$
\omega_{i}(\mathbf{x})=\alpha x_{i}+\alpha_{i}, \quad\left(i=1, \ldots, n ; \alpha^{\prime} \text { s are const. }\right)
$$

in an $\mathrm{n}$-dimensional domain $\Omega, \mathrm{n} \geq 2$, satisfying

$$
v \cdot \omega=1 \quad \text { on } \quad \Sigma=\partial \Omega
$$

where $v$ is the exterior unit normal on $\Sigma$, so we may construct a capillary surface $S: \quad u=u(x)$, defined over the base domain $\Omega$, such that $S$ meets vertical cylinder walls $z$ over $\Sigma=\partial \Omega$ in a constant angle $\gamma$ prescribed by $\cos \gamma<$ $\frac{1}{\operatorname{lub}_{\Omega}|\omega|}$, and then it has the constant mean curvature $H=\alpha \cos \gamma$.

Indeed, it is easily seen that this vector field (4.1) satisfies the conditions $(1.4),(1.5)$ and $(1.6)$, so we may consider this field related to the capillary surface

$$
u_{i}(x)= \pm \frac{\omega_{i} \cos \gamma}{\sqrt{1-|\omega|^{2} \cos ^{2} \gamma}}, \quad(i=1, \ldots, n)
$$

which is obtained from equation (2.1). Now, substituting (4.1) into (4.3), we find

$$
u_{i}(x)= \pm \frac{\left(\alpha x_{i}+\alpha_{i}\right) \cos \gamma}{\sqrt{1-\left[\left(\alpha x_{1}+\alpha_{1}\right)^{2}+\cdots+\left(\alpha x_{n}+\alpha_{n}\right)^{2}\right] \cos ^{2} \gamma}}, \quad\left(u_{i}=\frac{\partial u}{\partial x_{i}}\right)
$$

where the condition of integrability

$$
\frac{\partial u_{i}}{\partial x_{j}}=\frac{\partial u_{j}}{\partial x_{i}}, \quad(i, j=1, \ldots, n)
$$

is satisfied. Integrating (4.4), we obtain

$$
u(x)=\beta \mp \frac{1}{\alpha \cos \gamma} \sqrt{1-|\omega|^{2} \cos ^{2} \gamma}
$$

which is a sphere ( $\beta$ is a constant). 
ii) In general, if a vector field $\omega_{i}(x)$ satisfies the following two conditions with the constant div $\omega$,

$$
\left(1-|\omega|^{2} \cos ^{2} \gamma\right)\left(\frac{\partial \omega_{i}}{\partial x_{j}}-\frac{\partial \omega_{j}}{\partial x_{i}}\right)+\left(\cos ^{2} \gamma\right) \sum_{k=1}^{n}\left(\frac{\partial \omega_{j}}{\partial x_{k}} \omega_{i} \omega_{k}-\frac{\partial \omega_{i}}{\partial x_{k}} \omega_{j} \omega_{k}\right)=0 \quad(i, j=1, \ldots, n)
$$

in an $\mathrm{n}$-dimensional domain $\Omega, \mathrm{n} \geq 2$, and

$$
\mathrm{v} \cdot \omega=1 \text { on } \Sigma=\partial \Omega,
$$

then we can construct a capillary surface $S$, defined over the base domain $\Omega$, such that $S$ meets vertical cylinder walls $z$ over $\Sigma=\partial \Omega$ in a constant angle $\gamma$ prescribed by $\cos \gamma<\frac{1}{\operatorname{lub}|\omega|}$, and then it has the constant mean curvature $\mathrm{H}=\frac{\cos \gamma}{\mathrm{n}} \operatorname{div} \omega$.

Indeed, since equation (4.7) is the condition of integrability of equation (4.3) and the given vector field $\omega_{i}(x)$ also satisfies the conditions $(1.4),(1.5)$ and (1.6), the related capillary surface $s: u=u(x)$ can be found from equation (4.3).

ACKNOWLEDGMENT. This work was completed while the author was a visiting scholar at Stanford University. 


\section{REPERENCES}

1. FINN, R. Cap1llary Phenomena, Uspechi Mat. Nauk 29 (1974) 131-152.

2. FINN, R. Existence and Non-Existence of Capillary Surfaces, Manuscripta Math. 28 (1979) 1-11.

3. KILLING, W. Über die Grundlagen der Geometrie, J. Reine Angew. Math. 109 (1892) 121-186.

4. EISENHART, L. P. Riemannian Geometry, Princeton University Press, 1949.

5. EISENHART, L. P. Continuous Groups of Transformations, Princeton University Press, 1933.

6. YANO, K. Differential Geometry on Complex and Almost Complex Spaces, The Macmillan Co., 1965.

7. YANO, K. The Theory of Lie Derivatives and its Applications, Amsterdam, 1957.

8. HIRAMATU, H. On Some Properties of Groups of Homothetic Transformations in Riemannian and Finslerian Spaces, Tensor 4 (1954) 28-39. 


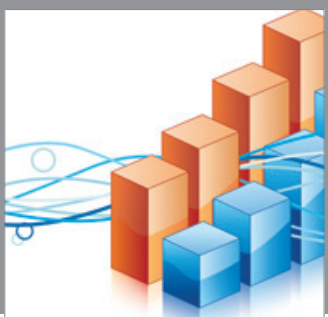

Advances in

Operations Research

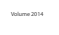

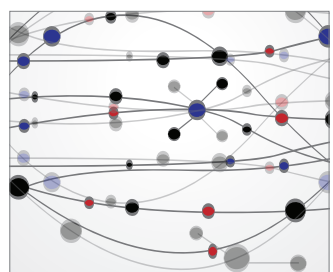

\section{The Scientific} World Journal
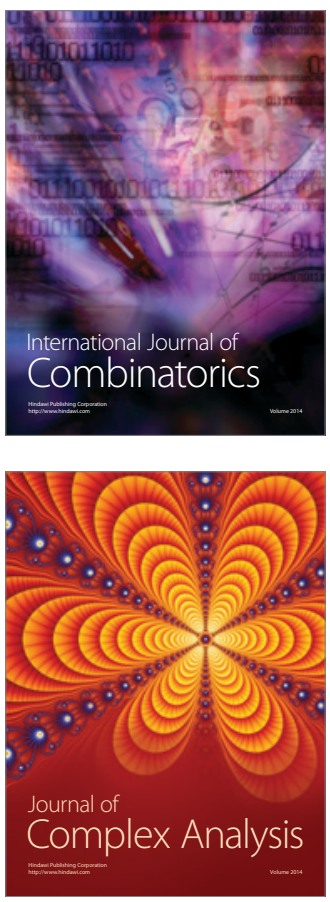

International Journal of

Mathematics and

Mathematical

Sciences
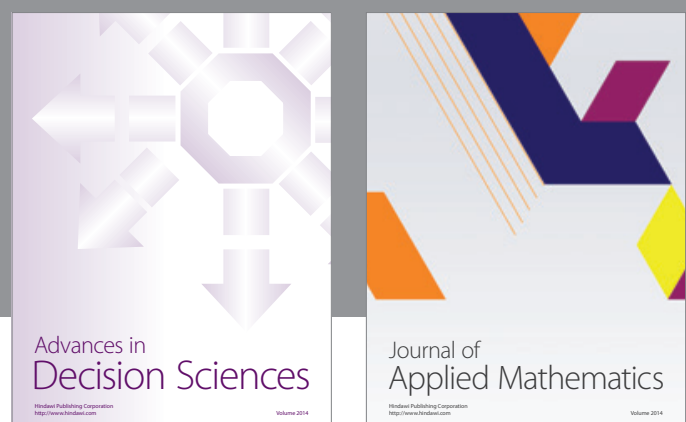

Journal of

Applied Mathematics
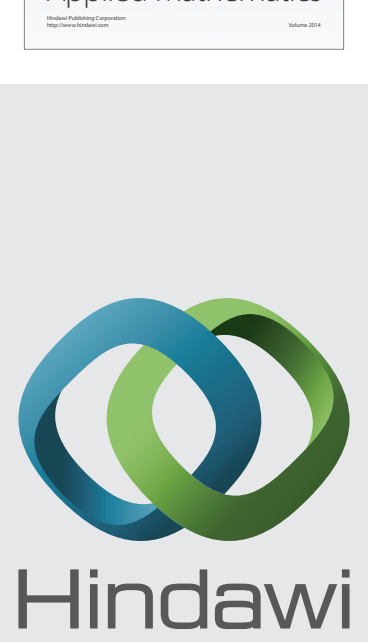

Submit your manuscripts at http://www.hindawi.com
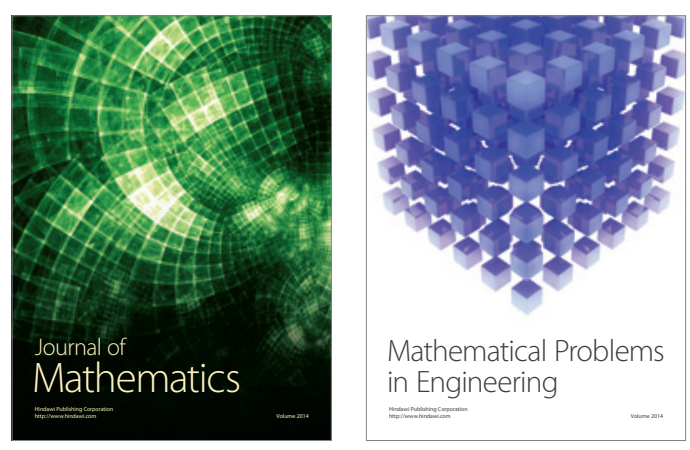

Mathematical Problems in Engineering
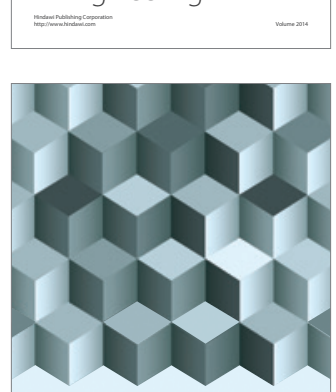

Journal of

Function Spaces
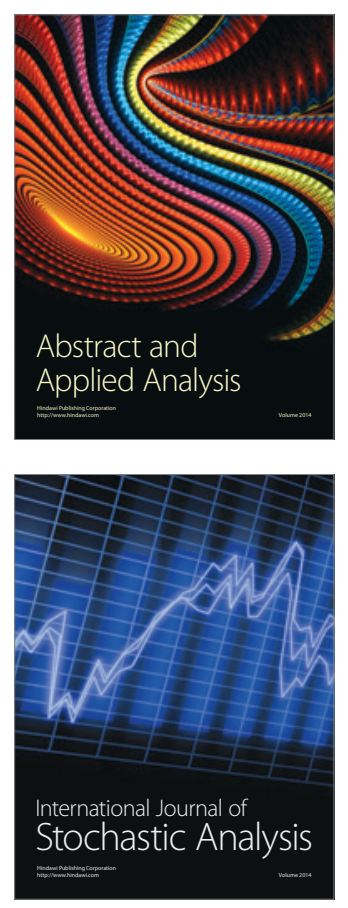

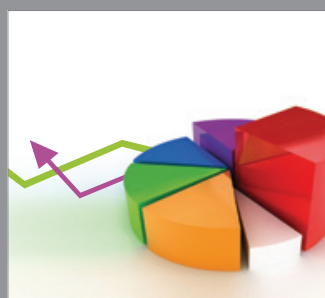

ournal of

Probability and Statistics

Promensencen
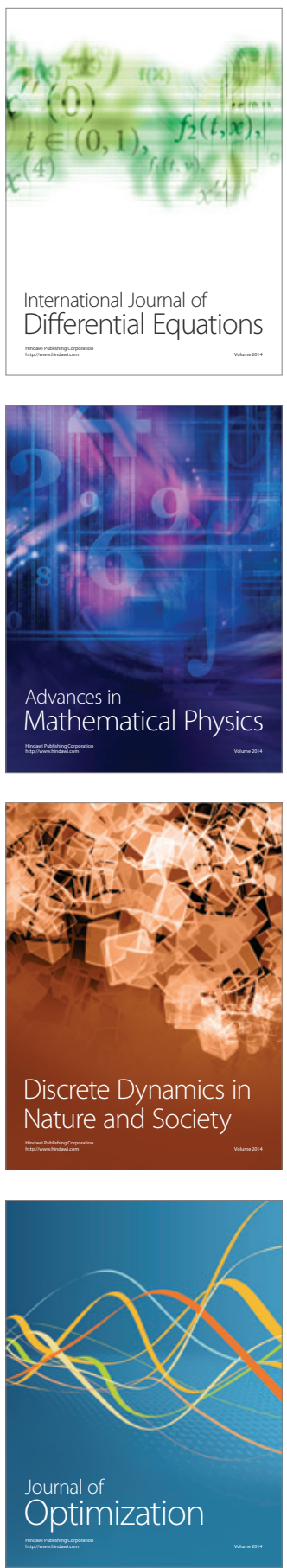